\title{
Second solar spectrum of the Sr I $4607 \AA$ Aine: depth probing of the turbulent magnetic field strength in a quiet region
}

\author{
M. Derouich ${ }^{1,2}$, V. Bommier ${ }^{2}$, J. M. Malherbe ${ }^{3}$, and E. Landi Degl'Innocenti ${ }^{4}$ \\ 1 Instituto de Astrofísica de Canarias, 38205 La Laguna, Tenerife, Spain \\ e-mail: moncef@iac.es \\ 2 Laboratoire d'Étude du Rayonnement et de la Matière en Astrophysique, CNRS UMR 8112 - LERMA, Observatoire de Paris, \\ Section de Meudon, 92195 Meudon, France \\ ${ }^{3}$ Laboratoire d'Études Spatiales et d'Instrumentation en Astrophysique, CNRS UMR 8109 - LESIA, Observatoire de Paris, \\ Section de Meudon, 92195 Meudon, France \\ 4 Università degli Studi di Firenze, Dipartimento di Astronomia e Scienza dello Spazio, Largo E. Fermi 2, 50125 Firenze, Italy
}

Received 14 December 2005 / Accepted 22 June 2006

\section{ABSTRACT}

\begin{abstract}
Aims. This paper is devoted to an interpretation of Quiet-Sun, spatially-resolved spectropolarimetric observations of the Hanle effect in terms of turbulent weak magnetic field determination.

Methods. Observations: the slit was positioned perpendicular to the limb, and the spatial resolution along the slit was 1 arcsec, leading to a depth probing along 132 different limb distances. The new polarimeter of the Pic-du-Midi Turret Dome was used on May 14, 2004 to observe a quiet region at the East limb equator in the resonance line of neutral Strontium at $4607 \AA$.

Results. For each limb distance, we properly adjusted the theoretical intensity profile obtained by applying a zero-field model to the observed one. Micro- and macroturbulent velocities were thus derived (average values $v_{\text {micro }}=1.77 \mathrm{~km} \mathrm{~s}^{-1}$ and $v_{\text {macro }}=1.95 \mathrm{~km} \mathrm{~s}^{-1}$ ). The magnetic field was determined in a second step by interpreting the Hanle effect on the line center linear polarization degree. The depolarizing collisions with neutral hydrogen were taken fully into account through a semi-classical calculation of their rates. An average value of $B=38$ Gauss was thus derived. Finally, error bars on the magnetic field values were evaluated from a) the polarimetric inaccuracy, b) the limb distance determination inaccuracy, and c) the uncertainty on our theoretical collisional depolarizing rates that we evaluated. This combination leads to $\sim 10-20 \%$ as total relative error on the magnetic field determination by the Hanle effect method. Since the inaccuracy due to the model itself was hard to properly evaluate, it was ignored. An uncertainty of $\pm 60 \mathrm{~km}$ on the line formation depth was, however, derived from the contribution functions. The magnetic field is found to increase slowly with height in the height range $220-300 \mathrm{~km}$ above $\tau_{5000}=1$ and then decrease in the height range $300-370 \mathrm{~km}$.
\end{abstract}

Key words. scattering - Sun: photosphere - Sun: magnetic fields - line: formation - polarization

\section{Introduction}

Close to the solar limb, absorption lines of scattering atomic systems (diatomic molecules and simple/complex atoms/ions) show linear polarization mainly because they are illuminated by anisotropic radiation. The spectrum of the linear polarization, which is conventionally called the "second solar spectrum", contains rich information about the anisotropies and weak magnetic fields in the solar atmosphere. This information is generally inaccessible by interpreting the ordinary intensity spectrum. In particular, interpreting the second solar spectrum in terms of Hanle effect is now the only way to study weak turbulent (i.e. spatially unresolved within the present observational possibilities) magnetic fields at the surface of the quiet Sun.

The SrI $4607 \AA$ is one of the most interesting polarized lines of the second solar spectrum for which the two-level atom model is justified. Several works are concerned with this line but are limited to the interpretation of the linear polarization degree observed without spatial resolution (e.g. Faurobert et al. 2001; Trujillo Bueno et al. 2004; and Bommier et al. 2005, hereafter Paper I). Our aim is to provide depth probing of the turbulent magnetic field strength by interpreting spectropolarimetric observations at 132 limb distances ranging from 1 to 132 arcsec.
In order to discuss whether the magnetic field strength varies within the range of heights of line-center formation, we carefully computed the error bars inferred from, a) the polarimetric inaccuracy, b) the limb distance determination inaccuracy, and c) the uncertainty on our theoretical collisional depolarizing rates that we evaluated. In the framework of our model, the Hanle effect technique permits us to determine the magnetic field within the global 10 to $20 \%$ inaccuracy.

Section 2 is devoted to describing of the theoretical background. The linear polarization is due to scattering, and the various depolarizing mechanisms that compete are introduced. Section 3 is devoted to the observations and data reduction. The method of interpretation is described in Sect. 4, and the accuracy on the results is evaluated in Sect. 5. The final results are given in Sect. 6, and all the results are summarized in the concluding Sect. 7.

\section{Competing depolarization mechanisms}

Since the $4607 \AA$ line is the resonance line of SrI, namely $5 \mathrm{~s}^{2}{ }^{1} \mathrm{~S}_{0} \rightarrow 5 \mathrm{~s} 5 \mathrm{p}^{1} \mathrm{P}_{1}$, we consider a two-level atom having a lower level with angular momentum $J=0$ and an upper level with angular momentum $J^{\prime}=1$ and Landé factor $g_{J^{\prime}}=1$. The 
states $5 \mathrm{~s}^{2}{ }^{1} \mathrm{~S}_{0}$ and $5 \mathrm{~s} 5 \mathrm{p}{ }^{1} \mathrm{P}_{1}$ are described by the spherical tensor components ${ }^{J} \rho_{q}^{k}$ and ${ }^{J^{\prime}} \rho_{q}^{k}$ of the density matrix, respectively. Because $J=0,{ }^{J} \rho_{0}^{0}$ is the only non-vanishing density-matrix element in the lower level. In contrast, the upper level with $J^{\prime}=1$ can be linearly polarized. Due to the symmetry of the turbulent field, in the limb reference frame the non-zero Stokes parameters are $Q$ and $I$, which are respectively associated to the tensorial ranks $k=2$ and $k=0$ ( $q=0$ due to symmetry). In fact, the second solar spectrum of $\operatorname{Sr}$ I $4607 \AA$ Stokes $U$ has always been found to be zero, leading to the unresolved field model that causes depolarization of $Q / I$ but no rotation of the plane of polarization.

In the coupled sets of statistical equilibrium and radiative transfer equations, the depolarizing effect of turbulent magnetic field is described by the Hanle factor $\mu_{k}(k=0,2)$ (e.g. see Paper I). To a very good approximation, one can assume that the intensity $I$ is not affected by the presence of a turbulent magnetic field $\left(\mu_{0}=1\right)$. However, one has $1 / 5<\mu_{2}<1$, which corresponds to a depolarization of the Sr I $5 \mathrm{~s} 5 \mathrm{p}^{1} \mathrm{P}_{1}$ level by the Hanle effect. This results from the fact that the magnetic field partially destroys the coherences between the $\left(2 J^{\prime}+1\right)$ Zeeman sublevels leading to a decrease in the line linear polarization $Q / I . \mu_{2}=1$ for the zero-field case. In the saturation regime reached for sufficiently high magnetic field values, $\mu_{2}=1 / 5$, and the theoretical linear polarization becomes insensitive to the Hanle effect.

In addition to the magnetic field effect, it is important to notice that collisions induce comparable modifications of the spherical tensor components ${ }^{J^{\prime}} \rho_{q}^{k}$. In the region of formation of the SrI $4607 \AA$ line, the isotropic elastic collisions of Sr I with hydrogen atoms play an important role in the alignment $(k=2)$ destruction of the SrI 5s 5p ${ }^{1} \mathrm{P}_{1}$ level. In fact, these collisions tend to reestablish the thermodynamical equilibrium between the Zeeman sublevels of the $5 \mathrm{~s} 5 \mathrm{p}^{1} \mathrm{P}_{1}$ level and to destroy their coherences so that atomic polarization vanishes. There is no saturation regime in the collisional depolarizing mechanism. The atomic levels become completely unpolarized if the isotropic collisions dominate any other polarizing effect. For example, the scattering polarization of Sr I $4607 \AA$, calculated in the absence of magnetic fields but in the physical conditions of the upper photosphere, decreases by about a factor 5 if the collisional depolarization rate is multiplied by 10 .

\section{Observations and data reduction}

As the instrument setup has already been decribed in great detail in Malherbe et al. (2006), we refer the reader to this paper and present only a summary of the observational procedure here.

We used the $50 \mathrm{~cm}$ refractor of the Pic du Midi Observatory, at $2870 \mathrm{~m}$ elevation, which was built in the sixties by J. Rösch with a new design (turret dome) to optimize image quality (the same concept was used in the eighties for the $2 \mathrm{~m}$ Bernard Lyot telescope). The turret dome is well known for the diffraction limited images of the solar granulation obtained in the G-band (around $4305 \AA$ ) by R. Müller ( 0.25 arcsec) (see Muller $\&$ Roudier 1984). In the eighties, Z. Mouradian from the Observatoire de Paris provided a powerful $8 \mathrm{~m}$ Littrow type spectrograph mounted on the equatorial mount just above the refractor. In 2004, we added to the instrument a nematic liquid crystal polarimeter located at the primary focus of the refractor. At that location, the beam has a cylindrical symmetry so that the instrument can be considered as polarization free. We used a variable retarder that is able to modulate sequentially between 0 to half wave in order to measure the Stokes parameter $Q$ relative to the linear polarization. We did not measure other parameters $(U$ and $V$ ). The modulation speed is about $50 \mathrm{~Hz}$ but is reduced by a factor 10 by the detector. The retardance can be exactly adapted to the wavelength of the observed line, so that the residual chromatism can be neglected (would not be the case with classical "achromatic" wave plates). The analysis was performed by a precision dichroïc linear polarizer, before the injection of the beam into the spectrograph.

We used this instrumental setup to measure the linear polarization of the SrI $4607 \AA$ line with the slit perpendicular to the West limb on May 14, 2004. As we were not far from the equator, we carefully checked the absence of active regions (we could not put the slit perpendicular to the limb at the poles because of built-in instrumental constraints). The CCD detector was an interline CCD with small full-well capacity (25000 electrons), 5 electron gain, and 8 electron readout noise, allowing very short exposure times $(50 \mathrm{~ms})$, which is very useful for freezing turbulence. We got 8000 couples of spectra $I+Q, I-Q$ within 90 min of observations, between 09:15 UT to 10:44 UT. The observation was followed by a dark current and a flat field at disk center (between 10:50 UT and 11:01 UT). The slit width and length were 0.6 arcsec and 150 arcsec, respectively. The digital sampling along the solar surface was 0.2 arcsec, which was reduced to 1 arcsec in the data processing in order to improve the signal-to-noise ratio. The sampling in the spectral direction was $11.3 \mathrm{~m} \AA$ per pixel.

The 16000 spectra were used for the data processing. It consists in two major steps. During the 90 min observation, the spectrograph exhibits slow and small mechanical shifts, so that we have to track the position of the line in wavelength, with a precision of about a few hundredths of spectral pixel. The location of the limb also has to be tracked with precision, as the linear polarization ratio $Q / I$ varies very fast when approaching the limb (increasing $Q$, decreasing $I$ ). We detected the limb position in the continuum close to the line and defined it as the inflexion point of the intensity curve as a function of limb distance. The precision of the method is about 1 to 2 arcsec. It depends on image quality, which was not so good during the observing run (no better than 1 arcsec).

The ratio $Q / I$ in the core of the $\mathrm{Sr} I$ line is displayed in Fig. 1 as a function of limb-distance. We have also plotted the intensity of the continuum close to the line whose inflection point indicates the limb position. The spatial resolution is 1 arcsec.

We also plotted the line profiles in Fig. 2 as a function of wavelength at 5, 10, 20, and 80 arcsec from the limb, corresponding to $\mu=0.10,0.14,0.20$, and 0.41 where $\mu$ is the cosine of the heliocentric angle $\theta$. The intensity and polarization data obtained at Pic-du-Midi (present data) are compared with those obtained at THEMIS (Paper I) in Table 1. It can be seen that the agreement is excellent, given the polarimetric accuracy $\pm 2 \times 10^{-4}$ at Pic-du-Midi determined in Fig. 1. Moreover, the value obtained at Pic-du-Midi at 5 arcsec, $p=1.192 \%$, fully agree with the one obtained by Gandorfer (2002).

It must be noticed that the continuum polarization was not introduced from a theoretical model, but deduced from observations. Flat-field data at disk center are used to determine the zero polarization level, as we know that there is a difference in the transmission of the polarimeter between 0 to half wave. This correction, derived from the flat field, is then applied to observations. But, as flat-field calibration and limb observations cannot be simultaneous, it may result in a small error (very difficult to estimate) in the measurement of the continuum polarization, due to small drifts of the instrumental setup. We preferred to use the 
Table 1. Observational data from THEMIS (Paper I) and Pic-du-Midi (present paper). From left to right: limb distance $d_{\text {limb }}$, cosine of the heliocentric angle $\mu$, ratio of line center intensity to the neighboring continuum intensity $I_{\text {cent }} / I_{\text {cont }}$ for both THEMIS and Pic-du-Midi, line center linear polarization degree $p(Q / I$ in the limb reference frame), for both THEMIS and Pic-du-Midi. For the three first limb distances 4,10 , and 20, there were two measurements at THEMIS on two different days.

\begin{tabular}{cccccc}
\hline \hline $\begin{array}{c}d_{\text {limb }} \\
\text { arcsec }\end{array}$ & $\begin{array}{c}\mu \\
\cos \theta\end{array}$ & $\begin{array}{c}I_{\text {cent }} / I_{\text {cont }} \\
\text { THEMIS }\end{array}$ & $\begin{array}{c}I_{\text {cent }} / I_{\text {cont }} \\
\text { Pic-du-Midi }\end{array}$ & $\begin{array}{c}p(\%) \\
\text { THEMIS }\end{array}$ & $\begin{array}{c}p(\%) \\
\text { Pic-du-Midi }\end{array}$ \\
\hline 4 & 0.091 & $0.647,0.642$ & 0.641 & $1.244,1.225$ & 1.242 \\
10 & 0.144 & $0.587,0.587$ & 0.594 & $0.959,0.869$ & 0.875 \\
20 & 0.203 & $0.554,0.558$ & 0.564 & $0.829,0.715$ & 0.690 \\
40 & 0.286 & 0.516 & 0.539 & 0.522 & 0.515 \\
80 & 0.400 & 0.503 & 0.521 & 0.325 & 0.366 \\
\hline
\end{tabular}

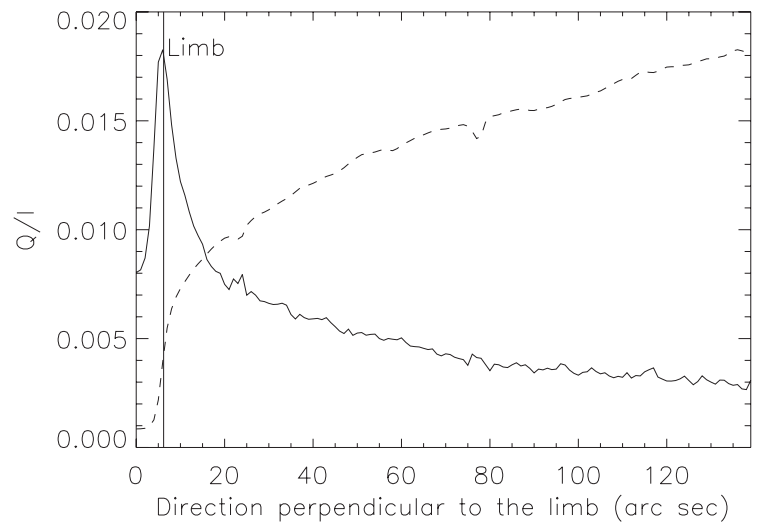

Fig. 1. Linear polarization ratio $Q / I$ as a function of limb distance $(\operatorname{arcsec})$ in the line core (solid line), together with the intensity of the continuum close to the line (dashed line). The location of the limb is indicated by the vertical bar.

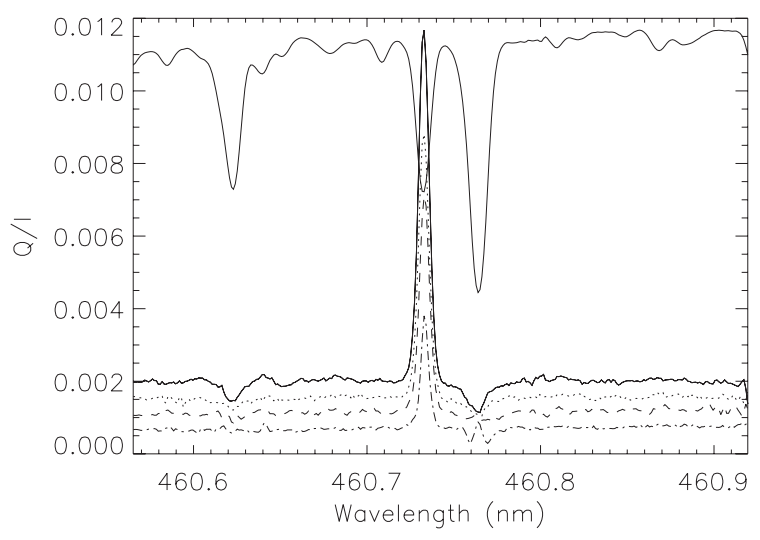

Fig. 2. Superimposed observations of $Q / I$ as a function of wavelength for four limb distances $5,10,20$, and 80 arcsec, the intensity profile is also reported in arbitrary units (solid line).

value given by the observational procedure rather than a theoretical one.

\section{Interpretation of the linear polarization observations in terms of turbulent weak magnetic field}

We consider the interpretation of the scattering polarization problem taking fully into account the main processes intervening in the polarized line formation, i.e. collisional interaction, magnetic field effect, and radiative processes (see Paper I for the theoretical formulation).
One of the differences with Paper I lies in the scattered light rate, which is now $r_{1}=10.3 \%$ in the notations of Paper I. The amplitude of this effect was determined as described in Paper I. Interestingly, accurate evaluation of this rate is of fundamental importance in determining the magnetic fields. Setting it, for instance, to zero leads to an overestimation of the magnetic field by approximately a factor two.

Besides, as stressed in Paper I, the magnetic field values are sensitive to the turbulent velocity values introduced in order to accurately model the intensity profile. Since the solar atmosphere is described by an empirical plane-parallel model, microturbulent and macroturbulent velocities have to be invoked as two free-fitting parameters to compute synthetic Stokes-I profiles at each limb distance. Because the light intensity is almost not affected by the Hanle effect, we start our analysis by accurately adjusting the computed intensity profile to the observed one in both depth and width, which allows determination of both microturbulent and macroturbulent velocities. For this purpose, at each of the 132 limb distances we built a basis of Stokes- $I$ profiles calculated for each couple $\left(v_{\text {micro }}, v_{\text {macro }}\right)$ ranging in the interval $\left[0.8 \mathrm{~km} \mathrm{~s}^{-1}, 3 \mathrm{~km} \mathrm{~s}^{-1}\right]$. We verified that a step size of $0.01 \mathrm{~km} \mathrm{~s}^{-1}$ is sufficient. Excellent agreement between computed and observed profiles is obtained at each limb distance for a unique pair of values $\left(v_{\text {micro }}, v_{\text {macro }}\right)$. We found average values of $v_{\text {micro }}=1.77 \mathrm{~km} \mathrm{~s}^{-1}$ and $v_{\text {macro }}=1.95 \mathrm{~km} \mathrm{~s}^{-1}$, in very good agreement with the ones determined with another instrument in Paper I.

To identify the height of line formation within the model atmosphere we applied the Eddington-Barbier approximation: it states that the line center forms at the height where $\tau / \mu=1$, with $\tau$ the line center optical depth along the vertical direction. For the model atmosphere used (same as in Paper I; temperature stratification from the Quiet Sun model by Maltby et al. 1986), the limb distances from 132 to 1 arcsec correspond to formation heights from 225 to $368 \mathrm{~km}$ above the $\tau_{5000}=1$ level.

The magnetic field strength is determined by introducing the 132 couples of velocities $v_{\text {micro }}$ and $v_{\text {macro }}$, together with the amplitude of scattered light into the model of the polarized line formation. The magnetic field value giving the smallest difference between theoretical and observed line center polarization degree was retained. The results of the magnetic field strength as a function of the measured limb distances are given in Fig. 3. In contrast, Fig. 4 displays the obtained magnetic field strength as a function of the height of the line-center formation derived from the atmospheric model. To point out trends in the depth dependence of the magnetic field, we applied the usual tools to attempt to reduce the field strength fluctuations by smoothing (Figs. 3 and 4). The turbulent magnetic field strength ranges from 29 to 46 Gauss with an average value of 38 Gauss. 


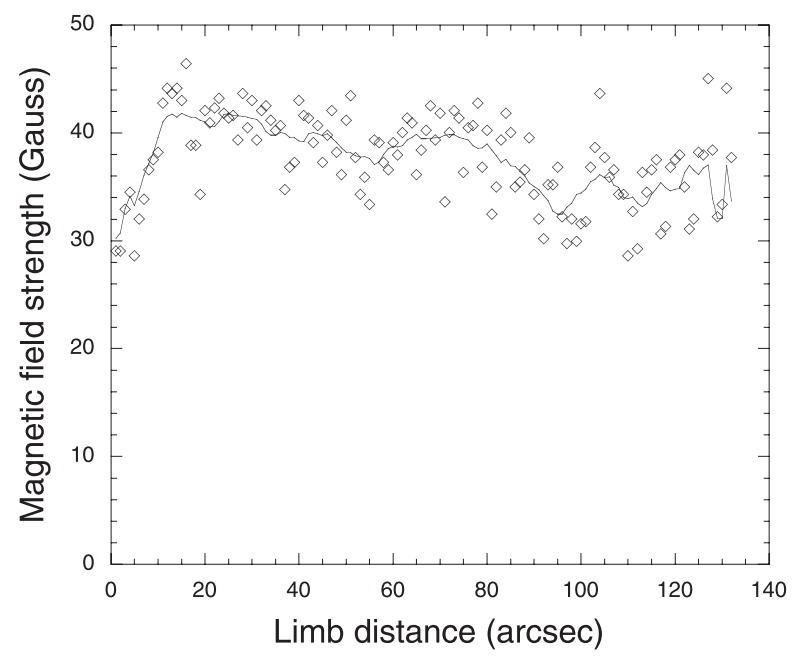

Fig. 3. Magnetic field strength in Gauss $(\diamond$-symbols) as a function of the limb distance. Full line: behavior of smoothed magnetic field fluctuations.

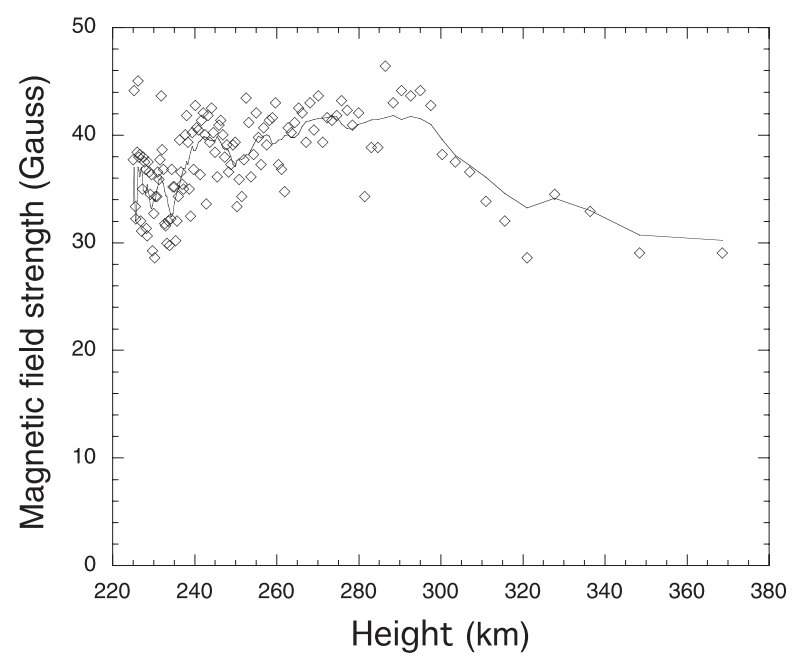

Fig. 4. Magnetic field strength in Gauss ( $\diamond$-symbols) as a function of the height of the line-center formation above the $\tau_{5000}=1$ level. Full line: behavior of smoothed magnetic field fluctuations.

\section{Discussion on the results accuracy}

In this section we discuss possible error sources and the accuracy of our results. In the following we take various origins of inaccuracy into account, as long as an evaluation is feasible.

\subsection{Model dependence}

Obviously, the fact of having used a plane-parallel model of line formation is responsible for inaccuracy due to the model itself, and in particular to its one-dimensional character.

First of all, the surface curvature was not taken into account: we think that the effect of surface curvature on the magnetic field diagnostic is probably very small or at least smaller than the other perturbing effects that we consider below. A second source of inaccuracy is the possible uncertainty in the atmospheric stratification itself. We employed the average Quiet Sun model atmosphere by Maltby et al. (1986). This model coincides with the FALC one of Fontenla et al. (1993 up to $1100 \mathrm{~km}$, but with a deeper extension up to $-451 \mathrm{~km}$. As this effect is very hard to properly evaluate, we discard it from the following evaluation.

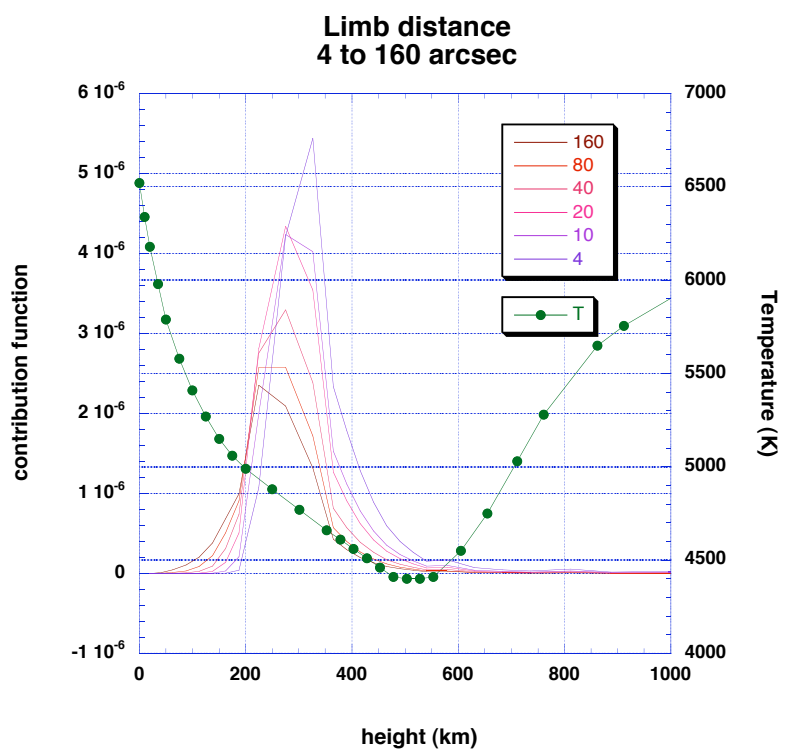

Fig. 5. Contribution functions for the emerging polarization (Stokes $Q$ ) of the Sr I $4607 \AA$ Aine (solid lines, left $y$-axis) and temperature (filled circles, right $y$-axis), as a function of the height above $\tau_{5000}=1$, for a series of limb distances ranging from 4 to 160 arcsec.

As for the model effect, it is however possible to plot the contribution function for the Stokes parameter $Q$ along the lineof-sight. A series of such contribution functions for various limb distances is plotted in Fig. 5. Each contribution function that we have plotted is the contribution of each depth interval to the integral (see for instance the contribution of each interval in Eq. (27) of Bommier et al. 1991). As apparent from the temperature curve, the depth points are not exactly equally spaced, so that the plotted contribution functions are only approximate, but sufficiently precise for the present analysis. From these contribution functions, we can estimate an uncertainty of $\pm 60 \mathrm{~km}$ for the height of the line center formation.

\subsection{Error bars}

In the following, we evaluate the error bars on the magnetic field determinations based on: a) polarimetric inaccuracy, b) uncertainty on the limb distance, and c) uncertainty on our theoretical collisional depolarizing rates.

From the noise along the curve in Fig. 1, we estimate the polarimetric inaccuracy on our $Q / I$ measurements to be $\pm 2 \times$ $10^{-4}$, which is not very far from the photon noise limit, which itself can be estimated as $\pm 0.5 \times 10^{-4}$ from the data given in Sect. 3. Similar uncertainties were obtained with the THEMIS observations of Paper I (see the uncertainties and photon noise level in Table 4 of Bommier \& Molodij 2002), but with a much lower number of limb distances (9 instead of 132), due to the low sensitivity of THEMIS in the blue part of the spectrum.

We estimate an uncertainty of \pm 1 arcsec for the limb distance. The determination of the limb position in the data reduction is described in Sect. 3. We take advantage of the fact that the limb position has been recorded when the slit was perpendicular to the limb.

The collisional rates are often a very important ingredient in a realistic analysis of the polarization regardless of modeldependent or independent Hanle-effect techniques. It important to assign a correct error bar to the determination of the collisional depolarization rate of an interesting line like Sr I $4607 \AA$. 
We adopted the collisional depolarizing rate $D^{2}$ of the Sr I $5 \mathrm{p}{ }^{1} \mathrm{P}_{1}$ level, which is calculated according to the general semi-classical theory developed in Derouich et al. (2003). The semi-classical $D^{2}$ can be written as a function of the local temperature $T$ and the neutral hydrogen density $n_{\mathrm{H}}$ :

$D^{2}=1.36 \times 10^{-8} \times n_{\mathrm{H}}\left(\frac{T}{5000}\right)^{0.374} \mathrm{~s}^{-1}$.

Estimating of the error bar on the semi-classical calculations of $D^{2}$ requires careful comparison with both experimental data and sophisticated quantum chemistry calculations. Unfortunately, there are no experimental values to compare with, so that we have to depend solely on quantum chemistry results. Quantum calculations of $D^{2}$ have been performed by FaurobertScholl et al. (1995) and, more recently, by Kerkeni (2002) who obtained a value about a factor of two smaller than FaurobertScholl et al. Something seems wrong, since using the same quantal approach means they should find almost same collisional rate. This disagreement and the fact that there is no experimental determination of $D^{2}$ complicate the situation when trying to estimate the error on the semi-classical calculations of $D^{2}$.

Generally speaking, for a given isoelectronic group, the larger the size of the perturbed atom, the higher the interaction potential energy (and consequently, the higher the depolarizing rate). It is surprising that this rule is not respected for the Sr I atom in Kerkeni (2002): the rate associated to the Sr I atom has the lower depolarizing rate if compared to the ones associated to the alkaline-earth elements $\mathrm{Mg} \mathrm{I}$ and $\mathrm{Ca} \mathrm{I}$.

To gain some understanding of the origin of the problem, one should keep in mind that the Sr I atom has the biggest size (38 electrons) compared to $\mathrm{Mg}$ I, $\mathrm{Ca}$ I, and $\mathrm{Na} \mathrm{I}$, which substantially complicates ab initio quantum calculations of the Sr I-H I interaction potential. In order to fix the error bar on our depolarizing rate of the $5 p{ }^{1} \mathrm{P}_{1}$ Sr I state, we decided to compare the semi-classical method (Derouich et al. 2003) adopted here to the quantal results of Kerkeni (2002) in the other available cases $\mathrm{MgI}, \mathrm{Ca}$ I, and $\mathrm{NaI}$, which have a small size and their quantum-chemistry study is not problematic. In fact, for these systems, the interaction potentials are extensively reviewed in the literature ${ }^{1}$, and the results are fairly consistent. At $T=$ $5000 \mathrm{~K}$, the difference between results of the general semiclassical theory (Derouich et al. 2003) and quantal results of Kerkeni (2002) is $11 \%$ for $\mathrm{MgI} 3 \mathrm{p}^{1} \mathrm{P}_{1}, 8 \%$ for CaI $4 p{ }^{1} \mathrm{P}_{1}$, and $13 \%$ for $\mathrm{Na}$ I $3 \mathrm{p}^{2} \mathrm{P}_{3 / 2}$.

Therefore, the mean inaccuracy with respect to quantum chemistry calculations is about $10 \%$. We think that it is reasonable to take $10 \%$ as expected error bar on the calculations of the semi-classical $D^{2}$ given by Eq. $(1)^{2}$.

In Fig. 6 we report the effect of each inaccuracy source on the magnetic field determination together with the effect of the total inaccuracy as a function of the line formation height. We note that, on average, the three sources of imprecision have comparable importance and that the total imprecision of these sources is $\sim 10$ to $20 \%$.

${ }^{1}$ See for example, Lemaire et al. (1985), Baird et al. (1979), Monteiro et al. (1988), Chambaud \& Lévy (1989), Anstee \& O'Mara (1991), Spielfiedel et al. (1991), Krsljanin \& Peach (1993), Leininger et al. (2000).

${ }^{2}$ It is normal that the most important difference (=13\%) occurs for the case of $\mathrm{NaI}$ because its fine structure interaction is neglected in Derouich et al. (2003). Unlike NaI, the SrI atom has no fine structure, which implies that our approach is certainly more accurate in the Sr I case.

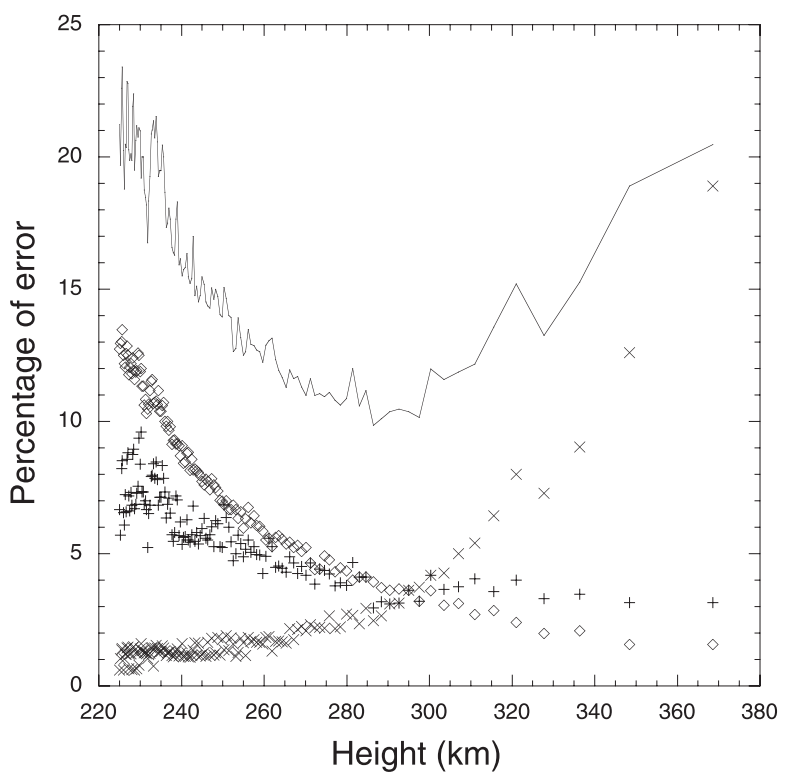

Fig. 6. Error sources as function of height. $\diamond$ : Relative error expressed in percent due to the effect of polarimetric sensitivity. + : Relative error due to the effect of eventual inaccuracy on the depolarizing rate $D^{2}$. $\times$ : Relative error on the measurement of the limb distance. Full lines: total relative error.

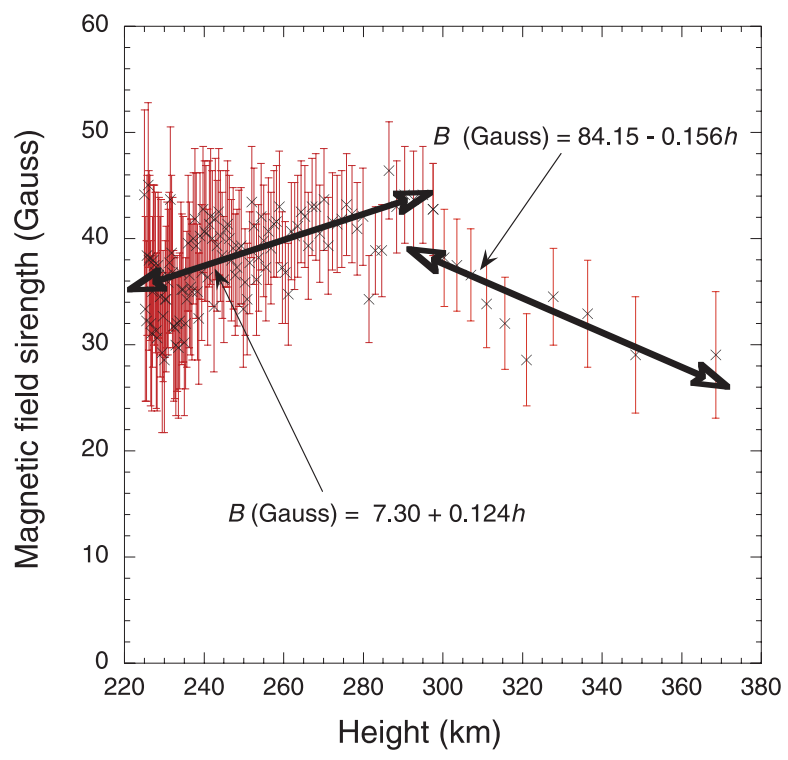

Fig. 7. Magnetic field as a function of the height of line formation with error bars. To describe the behavior of the field strength we draw two straight lines.

\section{Depth dependence of the magnetic field}

As reported in Fig. 7, we can draw two straight lines through the error bars. The first one represents a linear increase in the magnetic field below the height of the line-center formation $h \simeq$ $300 \mathrm{~km}$ (i.e. $11 \mathrm{arcsec}$ ) following the relationship

$B($ Gauss $)=7.81+0.122 h$

and the other one represents a linear decrease above $300 \mathrm{~km}$ :

$B($ Gauss $)=84.15-0.156 h$.

The average value is $B=38$ Gauss, in good agreement with the value $B=46$ Gauss determined without spatial resolution with 
THEMIS (see Paper I), if one considers the possible inaccuracies evaluated in the present paper. It has to be noted that the level of scattered light is $20 \%$ higher in the present observations than in the THEMIS observations of Paper I. In principle the effect of scattered light is fully taken into account in our analysis; however, we noticed that the higher the scattered light level, the lower the determined field strength, as we obtain here.

\section{Conclusion}

The Hanle effect is an interesting method for determining weak magnetic field within $\sim 10$ to $20 \%$ inaccuracy. In this inaccuracy evaluation, we have taken the following possible error sources into account: a) polarimetric inaccuracy, b) limb distance determination inaccuracy, and c) uncertainty on our theoretical collisional depolarizing rates. We ignored the inaccuracy due to the model itself being hard to evaluate properly.

Below $300 \mathrm{~km}$ above $\tau_{5000}=1$, the magnetic field strength appears to increase slowly with the height (derived from the line formation). In contrast, above $300 \mathrm{~km}$, the magnetic field displays a height-decreasing behavior. Its average value is $B=$ 38 Gauss, together with average velocity values of $v_{\text {micro }}=$ $1.77 \mathrm{~km} \mathrm{~s}^{-1}$ and $v_{\text {macro }}=1.95 \mathrm{~km} \mathrm{~s}^{-1}$.

In order to extend the diagnostics of turbulent magnetic fields over larger parts of the solar atmosphere, other quantitative interpretations of linearly polarized lines are needed that have different formation heights, also observed with spatial resolution.

Acknowledgements. M.D. gratefully acknowledges the partial support by the Spanish Ministerio de Educatión y Ciencia through project AYA2004-05792 and by the European Solar Magnetism Network. The authors would like to thank Dr. D.M. Fluri for useful suggestions and comments.

\section{References}

Anstee, S. D., \& O’Mara, B. J. 1991, MNRAS, 253, 549

Baird, J. P., Eckart, M. J., \& Sanderman, R. J. 1979 J. Phys. B, At. Mol. Phys., 12355

Bianda, M., Solanki, S. K., \& Stenflo, J. O. 1998, A\&A, 331, 760

Bommier, V., \& Molodij, G. 2002, A\&A, 381, 241

Bommier, V., Landi Degl'Innocenti, E., \& Sahal-Bréchot, S. 1991, A\&A, 244, 383

Bommier, V., Derouich, M., Landi degl'Innocenti, E., Molodij, G., \& Sahal-Bréchot, S. 2005, A\&A, 432, 295 (Paper I)

Chambaud, G., \& Lévy, B. 1989, J. Phys. B, At. Mol. Opt. Phys., 22, 3155

Derouich, M., Sahal-Bréchot, S., Barklem, P. S., \& O’Mara, B. J. 2003, A\&A, 404,763

Faurobert-Scholl, M., Feautrier, N., Machefert, F., Petrovay, K., \& Spielfiedel, A. 1995, A\&A, 298, 289

Faurobert, M., Arnaud, J., Vigneau, J., \& Frisch, H. 2001, A\&A, 378, 627

Fontenla, J. M., Avrett, E. H., \& Loeser, R. 1993, ApJ, 406, 319

Gandorfer, A. 2002, The Second Solar Spectrum: A high spectral resolution polarimetric survey of scattering polarization at the solar limb in graphical representation, Vol. 2, $3910 \AA$ to $4630 \AA$ (Hochschulverlag AG an der ETH Zurich)

Kerkeni, B. 2002, A\&A, 390, 791

Krsljanin, V., \& Peach, G. 1993, in Spectral Line Shapes (New York: Nova Science), 7, 527

Leininger, T., Gadéa, F. X., \& Dickinson, A. S. 2000, J. Phys. B, At. Mol. Opt. Phys., 33, 1805

Lemaire, J. L., Chotin, J. L., \& Rostas, F. 1985, J. Phys. B, At. Mol. Phys., 18, 95

Malherbe, J.-M., Moity, J., Arnaud, J., \& Roudier, Th. 2006, A\&A, submitted

Maltby, P., Avrett, E. H., Carlsson, M., et al. 1986, ApJ, 306, 284

Monteiro, T. S., Danby, G., Cooper, I. L., Dickinson, A. S., \& Lewis, E. L. 1988, J. Phys. B, At. Mol. Opt. Phys., 21, 4165

Muller, R., \& Roudier, Th. 1984, Sol. Phys., 94, 33

Spielfiedel, A., Feautrier, N., Chambaud, G., \& Lévy, B. 1991, J. Phys. B, At. Mol. Opt. Phys., 24, 4711

Trujillo Bueno, J., Shchukina, N., \& Asensio Ramos, A. 2004, Nature, 430, 326 